\title{
ESTRUCTURA Y DISTRIBUCIÓN ESPACIAL DE LA REPOBLACIÓN Y DIVERSIDAD DEL SOTOBOSQUE DE Hesperocyparis guadalupensis S. WATSON
}

\section{STRUCTURE AND SPATIAL DISTRIBUTION OF REPOPULATION AND UNDERSTORY DIVERSITY OF Hesperocyparis guadalupensis S. WATSON}

\author{
Israel de Jesús Gómez-Reyes ${ }^{1}$, J. Jesús Vargas-Hernández', F. Ofelia Plascencia- \\ Escalante $^{1}$, Luciana Luna-Mendoza ${ }^{2}$ y Marcos Jiménez-Casas ${ }^{1}$
}

\author{
'Colegio de Postgraduados, Postgrado en Ciencias Forestales, Montecillo, Texcoco, Edo. de México, México. ${ }^{2}$ Grupo de Ecología y Conservación de \\ Islas, A.C., Ensenada Baja California, México. \\ *Autor de correspondencia (fplascen@colpos.mx)
}

\section{RESUMEN}

Hesperocyparis guadalupensis S. Watson es un especie endémica de Isla Guadalupe en proceso de recuperación después de la erradicación de la cabra feral (Capra hircus). En 2019 se establecieron cinco sitios de muestreo (400 $\mathrm{m}^{2}$ ) en tres distintas coberturas de dosel: baja (CB, $\left.\leq 5 \%\right)$, media (CM, 6-23\%) y alta $(C A, \geq 24 \%)$ con el objetivo de evaluar su efecto sobre la dinámica de la repoblación y en la diversidad vegetal del sotobosque. Se obtuvo información de altura del árbol (A) y diámetro a la altura de pecho (DAP) de individuos juveniles (altura mínima de 1.5 y máxima de $10 \mathrm{~m}$ ) y de la diversidad del sotobosque; además, se establecieron cuatro sub-sitios de $1 \mathrm{~m}^{2}$ por sitio de muestreo, donde se registró el número de plántulas con altura menor de 1.5 $\mathrm{m}$. El nivel de cobertura del dosel no influyó en el número de juveniles, pero el número de plántulas fue mayor en $\mathrm{CA}(\mathrm{P}=0.003)$. Se identificaron tres categorías de altura $(2.5,4.5$ y $6.5 \mathrm{~m})$ y tres de diámetro $(2.5,7.5$ y $12.5 \mathrm{~cm})$ en la población; la mayor frecuencia de individuos se registró en las categorías menores (2.5 y $4.5 \mathrm{~m}$ de altura, 2.5 y $7.5 \mathrm{~cm}$ de diámetro), especialmente en CM y CA. En CB se encontró una distribución espacial uniforme $[L(t)<0]$, mientras que en CM y CA la distribución espacial fue agrupada $[L(t)>0]$. En el sotobosque se registraron 24 especies correspondientes a 21 géneros y 12 familias, sin diferencias estadísticas significativas entre las coberturas del dosel en riqueza diversidad alfa del sotobosque. Al menos $60 \%$ de las especies del sotobosque se comparten en las tres condiciones de dosel (diversidad beta). La estructura vertical y horizontal de juveniles y plántulas confirma la recuperación del bosque al mostrar un aumento de individuos en categorías bajas, con una distribución espacial asociada con la apertura de claros pequeños que fomentan la germinación y reclutamiento de nuevos individuos a la población.

Palabras clave: Hesperocyparis guadalupensis, distribución espacial, diversidad, estructura, repoblación natural, sotobosque.

\section{SUMMARY}

Hesperocyparis guadalupensis S. Watson is an endemic species of Guadalupe Island in the process of recovery after the feral goat (Capra hircus) eradication. In 2019 , five sampling sites $\left(400 \mathrm{~m}^{2}\right)$ were established on three different categories of canopy cover: low ( $L C, \leq 5 \%$ ), medium (MC, 6-23\%) and high ( $\mathrm{HC}, \geq 24 \%$ ) with the aim of evaluating the effect of canopy cover on the dynamics of regeneration and on the understory plant diversity. Information on tree height $(\mathrm{H})$ and diameter at breast height (DBH) was obtainded from juvenile individuals (minimum heights of 1.5 and maximum of $10 \mathrm{~m}$ ) and plant diversity of the understory was recorded; in addition, four sub-sites of $1 \mathrm{~m}^{2}$ were established per sampling site, where the number of seedlings with height less than $1.5 \mathrm{~m}$ was recorded. The canopy cover level did not influence the number of juveniles, but the number of seedlings was higher in $\mathrm{HC}(\mathrm{p}=0.003)$ Three categories of $\mathrm{H}(2.5,4.5$ and $6.5 \mathrm{~m})$ and three of $\mathrm{DBH}(2.5,7.5$ and 12.5 $\mathrm{cm}$ ) were identified in the population; the highest frequency of individuals was registered in the smallest categories $(2.5$ and $4.5 \mathrm{~m}$ in height, 2.5 and $7.5 \mathrm{~cm}$ in diameter), especially in MC and HC. At LC a uniform spatial distribution was found $[L(t)<0)]$, while at MM and $H C$ the spatial distribution was grouped $[(L(t)>0]$. In the understory, 24 species of 21 genera and 12 families were recorded, with no significant statistical differences between canopy covers in richness and alpha diversity of the understory. At least $60 \%$ of the understory species were shared among the three canopy conditions (beta diversity). The vertical and horizontal structure of juveniles and seedlings confirms the recovery of the forest by showing an increase of individuals in low categories, with a spatial distribution associated with the opening of small clearings that promote the germination and recruitment of new individuals to the population.

Index words: Hesperocyparis guadalupensis, diversity, natural repopulation, spatial distribution, structure, understory.

\section{INTRODUCCIÓN}

Hesperocyparis guadalupensis S. Watson es un árbol de 15 a 20 m de altura, con fustes de 30-60 cm (Garcillán et al, 2009), endémico de Isla Guadalupe, Ensenada, Baja California, México, e incluido en la NOM-059SEMARNAT-2010 (SEMARNAT, 2010), localizado en la parte norte de la Isla, junto con el bosque de pino (Pinus radiata D. Don.), cubrían una superficie de 650 y 950 ha respectivamente (Oberbauer, 2006).

El intenso pastoreo por cabras (Capra hircus) por cerca de 200 años alteraron de manera notable los ecosistemas de la isla, considerados como frágiles (Junak et al., 2005), ocurriendo una reducción del 85 \% en la superficie ocupada por el ciprés; en 2006 y 2010 se reportaron 140-150 ha (Oberbauer 2006, Garcillán 2010). Además de las cabras, la invasión de plantas introducidas ha sido extensa en la 
isla, teniendo también un efecto negativo en las especies endémicas (Junak et al., 2005). Las cabras ferales fueron erradicadas en 2007 (Luna-Mendoza et al. 2019); a partir de esta erradicación, las especies nativas han recuperado terreno frente a plantas invasoras, principalmente la avena (Avena sp.) (Ceceña-Sánchez et al., 2018).

A pesar de que el bosque de ciprés ha iniciado su recuperación, existen pocos estudios que documenten esta dinámica después de la erradicación de las cabras, así como de la estructura vertical y distribución espacial de la repoblación y su relación con la diversidad vegetal en el sotobosque. Estos componentes y procesos de la comunidad vegetal proporcionan información sobre la productividad del ecosistema y su capacidad de recuperación y resiliencia posterior a la perturbación ocasionada por el sobrepastoreo y la invasión de especies exóticas invasoras (Ruiz-Jaén y Aide, 2005). Los objetivos del presente estudio fueron determinar la densidad de plántulas y juveniles establecidos, calcular el índice de distribución espacial de la repoblación, describir la estructura poblacional de la especie y determinar la diversidad vegetal del sotobosque en un rodal de $\mathrm{H}$. guadalupensis bajo diferentes coberturas de dosel.

\section{MATERIALES Y MÉTODOS}

\section{Área de estudio}

La Isla Guadalupe es una isla de origen volcánico, formada hace aproximadamente 7 millones de años (Moran, 1996) y es parte de la Reserva de la Biosfera Isla
Guadalupe, ubicada en el Océano Pacifico, a 260 km de la costa de Baja California, México (CONANP, 2013). El estudio se realizó en el rodal central (no fue afectado por el incendio de 2008) del bosque de ciprés, cerca de la zona llamada "Monte Augusta" (1298 msnm, la parte más alta de la Isla) (Figura 1), ubicado entre las coordenadas $29^{\circ}$ $06^{\prime} 37.16^{\prime \prime} \mathrm{N}^{\prime}$ y $29^{\circ} 05^{\prime} 14.24^{\prime \prime} \mathrm{N}$, y $118^{\circ} 18^{\prime} 50.15^{\prime \prime} \mathrm{O}$ y $118^{\circ}$ $20^{\prime} 9.80^{\prime \prime} \mathrm{O}$, con una superficie de 9 ha aproximadamente (Garcillán et. al., 2009).

El rodal presenta árboles adultos e individuos de diferentes edades de $H$. guadalupensis que se han establecido en zonas con distinta cobertura de dosel, posterior a la erradicación de las cabras. El rodal de estudio se encuentra a una altitud aproximada de 1200 m, exposición oeste y una pendiente menor de $10 \%$. No se cuenta con registros de precipitación media anual, ni de temperatura en particular para el rodal. El clima de la isla es de tipo mediterráneo, de acuerdo con la clasificación climática de Köppen modificada por García (2004), el clima en las costas es tipo BS, con subtipos mediterráneos templados, BSKs con temporada de lluvias en invierno. La parte noroeste de la isla se caracteriza por la presencia de neblinas constantes y una mayor precipitación que la zona del extremo sur. A nivel de la isla se reporta una precipitación media anual de $133 \mathrm{~mm}$ y temperatura media anual que varía entre 16.2 y $20.4^{\circ} \mathrm{C}$ (Garcillán et al., 2009).

\section{Cobertura del dosel}

Para la estimación del nivel de cobertura del dosel se utilizaron tres métodos. El primero de ellos se realizó a
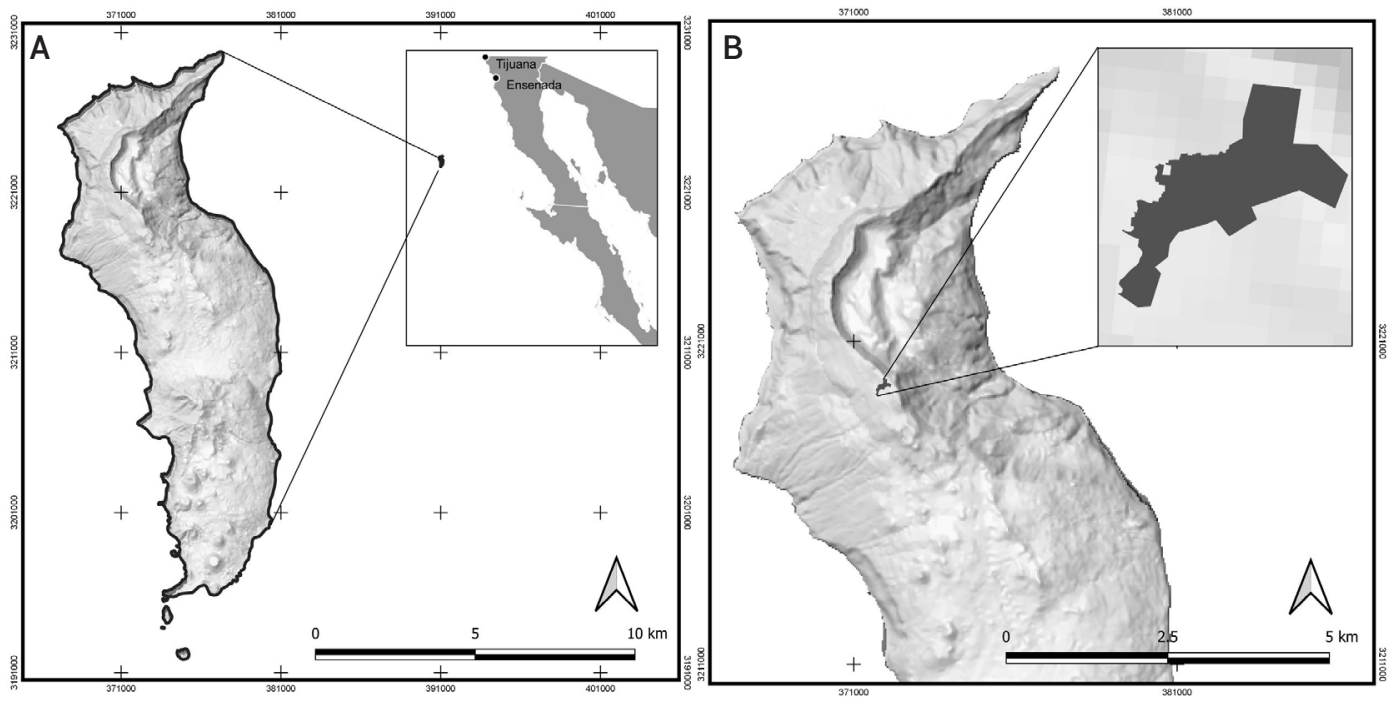

Figura 1. (A) Localización geográfica de Isla Guadalupe, México, (B) ubicación del área de estudio (rodal de $H$. guadalupensis). 
partir de una imagen satelital QuickBird Digital Globe con resolución espacial $2.44 \mathrm{~m}$ en escala multiespectral del año 2005; el segundo se derivó por medio de imágenes obtenidas con un Vehículo Aéreo No Tripulado (VANT) en 2019, procesadas con el software PIX4Dmapper, versión de prueba, para obtener el ortomosaico correspondiente; las imágenes fueron georreferenciadas en campo y corregidas. El tercer método se realizó directamente en campo, se midió el diámetro de copa de los árboles y mediante el software QGIS se elaboraron mapas de la proyección de éstas para cada árbol. Las proyecciones de copa fueron corregidas para evitar traslapes y evitar sobreestimación. Los tres métodos de estimación de la cobertura del dosel mostraron alta correlación $(r \geq 0.841)$. Se utilizaron los datos con base en el diámetro y proyección de copas para los análisis cuantitativos (tercer método). Los niveles de cobertura se clasificaron en baja $(\mathrm{CB}, \leq 5 \%)$, media ( $\mathrm{CM}$, $6-23 \%$ ) y alta (CA, $\geq 24 \%)$.

\section{Establecimiento de los sitios de muestreo y variables medidas}

De acuerdo con la metodología utilizada por ToledoLópez et al. (2012), sugerida por Corral-Rivas et al. (2013) y modificada por Ventura-Ríos et al. (2017); en cada nivel de cobertura del dosel se establecieron cinco sitios circulares de $400 \mathrm{~m}^{2}$. La ubicación de cada sitio de muestreo en cada categoría de cobertura del dosel se realizó de manera aleatoria mediante una malla de puntos a cada 50 m (Corral-Rivas et al. 2013). Se georreferenció (GPS) el centro de cada sitio, y se registró la altitud, la exposición y la pendiente. En estos sitios se midió el diámetro normal $(\mathrm{DN}, \mathrm{cm})$ y altura $(\mathrm{H}, \mathrm{m})$ de todos los individuos adultos (individuos que por sus características de tamaño del diámetro y su corteza era evidente que estaban presentes desde antes de la erradicación de las cabras) y juveniles (individuos con altura mayor o igual a $1.5 \mathrm{~m}$ y máxima de 10 m). El diámetro de copa (DC, m) para adultos se estimó con base en el promedio de la proyección de la copa en dirección norte-sur y este-oeste. La localización espacial de los juveniles se determinó con base en el azimuth y la distancia horizontal $(\mathrm{m})$ de cada árbol al centro del sitio para establecer sus coordenadas cartesianas $(x, y)$ en el sitio, información necesaria para determinar el patrón de distribución espacial de los individuos juveniles; a la vez, se registró la densidad de juveniles y de especies arbustivas en cada sitio. En sub-sitios de $1 \mathrm{~m}^{2}$, uno por punto cardinal dentro de cada sitio de $400 \mathrm{~m}^{2}$ (Pommerening y Grabarnik, 2019), se registró el número de plántulas con altura menor a 1.5 m. (Toledo-López et al., 2012), modificado por los autores; además, se midió el porcentaje de cobertura y altura $(\mathrm{cm})$ de la avena y el número y abundancia de herbáceas para determinar los índices de diversidad alfa y beta.

\section{Análisis estadístico}

Las variables número de individuos juveniles y plántulas se analizaron con análisis de varianza utilizando el paquete estadístico R (R Core Team, 2019), usando como fuentes de variación, los niveles de cobertura del dosel. Se realizó un análisis de correlación de Pearson para establecer el nivel de asociación entre el nivel de cobertura y el número de individuos juveniles y plántulas. La estructura vertical y horizontal se determinó con base en las frecuencias por categorías de altura y diámetro. La homogeneidad de la distribución de frecuencias entre las categorías de cobertura del dosel se evaluó con una prueba de Ji-cuadrada. El patrón de distribución espacial se determinó con base en el índice $K_{(t)}$ de Ripley (Ripley, 1977). Posteriormente, $K_{(t)}$ se transformó a $L_{(t)}$ a través de la raíz cuadrada de la función propuesta por Besag (1977), la cual facilita la interpretación gráfica del índice. $K_{(t)}$ y $L_{(t)}$ se estimaron con un nivel de significancia $P \leq 0.01$ mediante simulaciones de Monte Carlo (Besag, 1977), con el software TOOL-BOX (Fisher, 2000).

Con los datos de especies arbustivas y herbáceas del sotobosque se construyeron curvas de especie-área (Greig-Smith, 1983) utilizando el software PAST 4.02 (Hammer et al., 2020). Se estimó el número esperado de especies con base en el esfuerzo de muestreo usando los estimadores Chao 1 (Chao, 1984), ACE (Chao y Lee, 1992) y Bootstrap (Smith y Van Belle, 1984). La diversidad alfa se obtuvo con los índices de riqueza de Margalef, ShannonWiener, Simpson (Moreno, 2001) y Fisher (Zarco-Espinoza et al., 2010). Debido a que los datos no cumplieron con la condición de normalidad, se utilizó la prueba de KruskalWallis para comparar el valor de los índices en los tres niveles de cobertura del dosel. Los índices de Jaccard y de Sørensen (Moreno, 2001) fueron estimados para calcular la diversidad beta. Los índices de diversidad vegetal para las especies herbáceas medidos en los sub-sitios $\left(4 \mathrm{~m}^{2}\right)$ se extrapolaron a $400 \mathrm{~m}^{2}$. La avena, especie introducida, no se incluyó en los índices de diversidad vegetal, se presenta por separado.

\section{RESULTADOS Y DISCUSIÓN}

\section{Número de juveniles y plántulas de $H$. guadalupensis establecidos}

El análisis de varianza no mostró diferencias significativas entre las categorías de cobertura para el número de juveniles. Los sitios de muestreo dentro de una misma categoría de cobertura del dosel presentaron una amplia variación, al igual que el número de juveniles, la correlación no fue significativa entre éstas $(r=-0.185$, Figura 2); en contraste, la cobertura del dosel influyó en el 
número promedio de plántulas, con un valor promedio 3.5 \pm 1.0 ind. $\mathrm{m}^{-2}$ en la CA; ésta fue estadísticamente diferente con respecto al resto de las cuberturas del dosel y presentó una alta correlación ( $r=0.918)$ (Figura 2).

El número promedio de juveniles estimado en todo el rodal fue de $773 \pm 326$ ind. ha ${ }^{-1}$, con una variación desde 55 (CB) hasta 1165 (CM) individuos ha-1, lo que refleja la alta heterogeneidad espacial en el rodal; en cambio, el número promedio de plántulas fue de $1.38 \pm 0.52 \mathrm{~m}^{-2}$ y varió de 0 (CB) a 3.5 (CA) individuos $\mathrm{m}^{-2}$. Los resultados se reportan en diferentes escalas para juveniles y plántulas debido al muestreo realizado, donde se utilizaron sub-sitios de dos diferentes superficies (400 y $1 \mathrm{~m}^{2}$ respectivamente). Sólo los juveniles contabilizados en sitios de $400 \mathrm{~m}^{2}$ se extrapolaron a individuos por hectárea. Estos resultados contrastan con algunas referencias que consideran a $H$. guadalupensis como especie pionera que requiere espacios abiertos para su establecimiento. Garcillán et al. (2009) mencionaron que el reclutamiento es mayor en zonas donde existe interacción con la cobertura de dosel.

Los resultados del estudio muestran un comportamiento distinto de los juveniles y las plántulas (Figura 2). La mayor cantidad de juveniles se encontraron en sitios con una cobertura de dosel entre 10 y $30 \%$ (Figura 2A) y la densidad de plántulas aumentó ligeramente conforme se incrementó la cobertura del dosel (Figura 2B); lo que sugiere que la repoblación de esta especie en su etapa inicial se beneficia de las condiciones que se generan bajo el dosel o copa del arbolado, las cuales pueden estar asociadas con mayor disponibilidad de humedad en el suelo, protección contra temperaturas extremas, vientos y mayor disponibilidad de nutrimentos. Hesperocyparis guadalupensis es considerada una especie mucho más vulnerable a la sequía que otras en la Isla (León et al., 2003). Por otro lado, la mayor densidad de plántulas bajo el dosel de árboles progenitores puede estar asociada con una limitada capacidad de dispersión de la especie, ya que sus semillas se acumulan debajo de las copas de árboles reproductivos.

Pocos estudios han documentado el número de plántulas de $H$. guadalupensis en Isla Guadalupe. Junak et al. (2005) destaca que en los años 2001-2003, en sus visitas a la Isla, nunca observaron plántulas de ciprés. Para 2004 apareció "un gran número de plántulas en diferentes sitios, principalmente cerca de las orillas de las arboledas" y en 2005 "más plantas fueron vistas"; los individuos observados en esos años presentaban una altura mayor a $10 \mathrm{~cm}$ y dos individuos de aproximadamente 2 m. Aguirre-Muñoz et al. (2011) estimaron entre 457 y 1100 individuos ha-1 en áreas afectadas y no afectadas por el incendio de 2008,

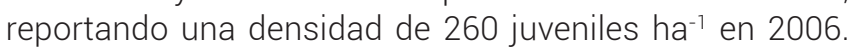
En el presente estudio se estimaron 773 juveniles ha-1 y 1.38 plántulas $\mathrm{m}^{-2}\left(13,800 \mathrm{ha}^{-1}\right)$, mostrando un aumento en el reclutamiento de nuevos individuos a la población con respecto a estudios anteriores. Los resultados del presente estudio evidencian la recuperación del bosque de ciprés, a pesar de los diferentes métodos de muestreo y criterios para clasificar los nuevos individuos usados en estudios previos.
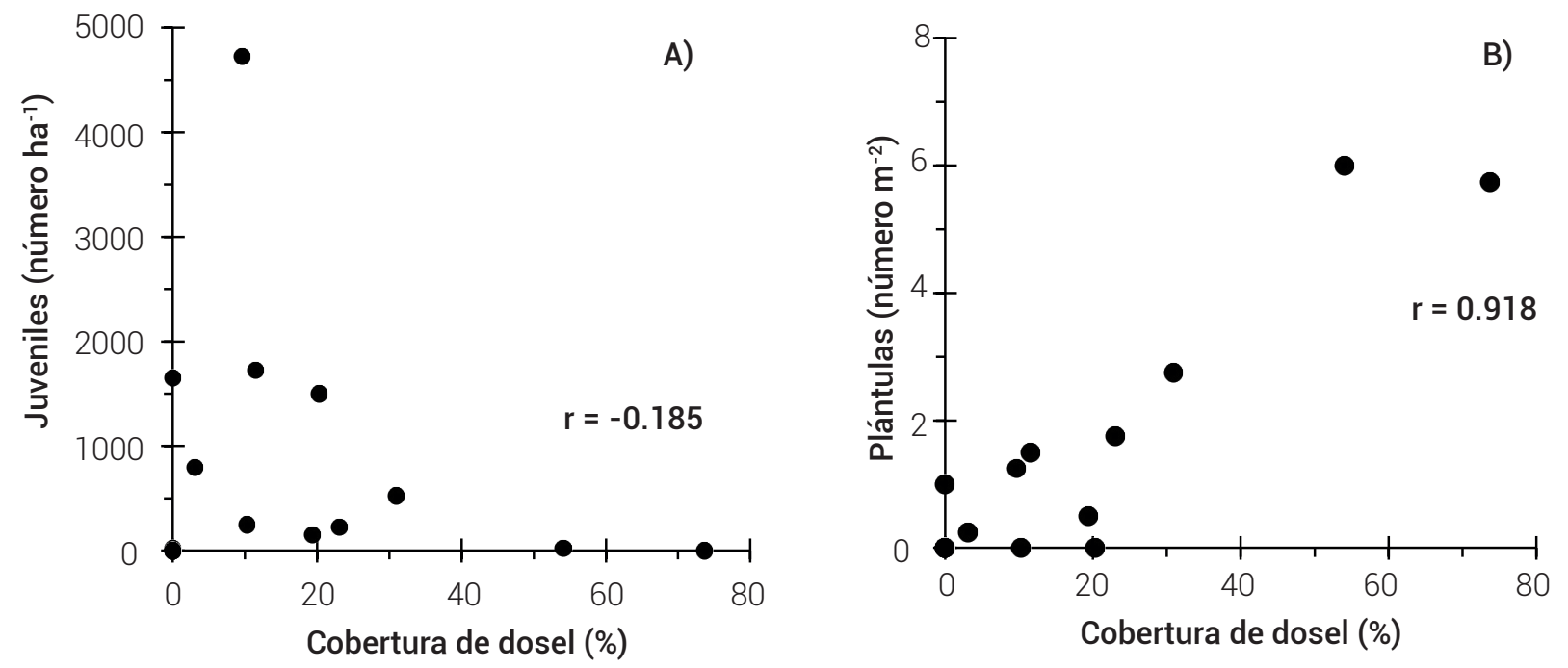

Figura 2. Relación del número de individuos juveniles ha-1 (A) y número de plántulas $\mathrm{m}^{-2}(\mathrm{~B})$ de $\mathrm{H}$. guadalupensis con la cobertura de dosel en un rodal en recuperación en Isla Guadalupe, México. 
Ustin et al. (1984), y Way y Pearcy (2012) sugirieron que para algunas especies los claros grandes reducen la germinación de la semilla y supervivencia de los plántulas; además, la tasa de reclutamiento puede aumentar cuando las plántulas están expuestas a periodos cortos de luz bajo doseles densos, ya que proporcionan a las plántulas condiciones menos extremas de temperatura y amortiguan los vientos. En algunos casos, esta característica puede indicar que la especie es tolerante a la sombra; sin embargo, no existe evidencia contundente de que $H$. guadalupensis sea una especie tolerante. Las condiciones bajo el dosel podrían explicar el mayor número de plántulas en CA y de juveniles en CM y CA. Garcillán et al. (2009) sostienen que en el bosque de ciprés la germinación ocurre principalmente dentro de los parches y en los bordes, en la zona más próxima a la caída de la semilla, pero la mortalidad es mayor en el interior, por lo que el reclutamiento se presenta principalmente en las márgenes. En el presente estudio se muestra una relación positiva entre el número de plántulas y la cobertura del dosel, la cual no se observa con los juveniles. Otros factores que pueden influir en el establecimiento de las plántulas es la profundidad de la capa de materia orgánica y la cobertura de vegetación herbácea, pero no se encontraron diferencias significativas entre las categorías de cobertura del dosel en la capa de materia orgánica $(1.4,2.2$ y $2.5 \mathrm{~cm}$ en $\mathrm{CB}, \mathrm{CM}$ y $\mathrm{CA}$, respectivamente) ni en la presencia de avena (21.3, 19.8 y $20.7 \%$, respectivamente).

\section{Estructura y distribución espacial de la repoblación de $H$. guadalupensis}

Se identificaron cuatro categorías de altura y diámetro en los individuos juveniles (Figura 3 ). En las categorías menores para altura ( 2.5 y $4.5 \mathrm{~m}$ ) y diámetro $(2.5$ y $7.5 \mathrm{~cm})$ se registró el mayor número de individuos, especialmente en CA y CM, ésto indica que la repoblación tiende a ocurrir en zonas con cierta protección durante las primeras etapas de desarrollo; posteriormente, requieren menos influencia directa de los adultos (Garcillán et al., 2009). El menor número de individuos en CB modificó ligeramente la estructura vertical y horizontal en esta condición de cobertura del dosel con respecto a las otras dos, pero la prueba de Ji-cuadrada mostró una distribución de frecuencias homogénea en las tres condiciones.

La distribución de las clases de altura y diámetro de los juveniles de $H$. guadalupensis muestran incorporaciones recientes y sucesivas, con un mayor número de individuos en las clases más pequeñas, lo que sugiere que la especie requiere condiciones de protección durante los primeros años de crecimiento, y explicaría el bajo número de juveniles en la cobertura del dosel baja; sin embargo, para poder crecer en altura, los individuos establecidos requieren luz directa. Esta característica se ha observado en Abies religiosa, que obtiene protección de factores ambientales adversos por parte de un dosel arbustivo durante sus primeras etapas de desarrollo (RodríguezLaguna et al. 2015) .

El patrón de distribución espacial de los juveniles, de acuerdo con los valores de la función $K_{(t)}$ de Ripley, fue muy heterogénea. En CB sólo se encontraron plántulas en uno de los sitios, con una distribución espacial uniforme a cualquier distancia $\left(L_{(t)}<0\right)$ (Figura $\left.4 A\right)$. En los sitios CM (Figura 4B) y CA (Figura 4C) la distribución espacial de los juveniles fue agrupada $\left(L_{(t)}>0\right)$. Las diferencias en la distribución espacial pueden atribuirse a diferencias en el número de árboles maduros encontrados en cada cobertura, especialmente en $\mathrm{CB}$; es posible que la disponibilidad de semilla en CB sea menor, lo que disminuye la posibilidad de establecimiento de plántulas. Garcillán (2010) mencionó que el reclutamiento de nuevos individuos de $\mathrm{H}$. guadalupensis se presenta de forma especialmente abundante alrededor de árboles recién caídos.

Los índices de distribución espacial agrupados son comunes en la repoblación de especies arbóreas; por ejemplo, las plántulas de Abies spp. generalmentepresentan una distribución espacial agrupada, como resultado de la apertura de claros en el dosel o por disminución (densidad) de follaje del dosel (Plateros-Gastélum et al., 2018; Spies, 1998; Ustin et al., 1984). En este estudio se observaron algunos claros en CM y CA ocasionados por la caída de árboles, lo que coincide con las afirmaciones hechas por estos autores, donde el principal factor que se favorece con la formación de claros en el dosel es la mayor entrada de luz hacia la parte baja. Maciel-Nájera et al. (2020) analizaron patrones espaciales de áreas repobladas naturalmente en bosques de pino-encino en la Sierra Madre Occidental (norte de México) en relación con tres factores: la pendiente, la exposición y la distancia entre cada árbol joven hasta el borde del rodal, encontraron patrones espaciales atípicos de la repoblación natural en rodales incoetáneos de pino-encino, atribuidos a un efecto nodriza del dosel, ya que la repoblación del complejo de especies analizado se estableció preferencialmente en las orillas de los claros. Este patrón es similar a lo observado en la repoblación natural de $H$. guadalupensis.

\section{Diversidad del sotobosque de $H$. guadalupensis}

Se encontraron 17 especies en CB, 16 en CM y 17 en CA con base en los indicadores S(est), ACE Mean y Chao de las curvas área-especie (Figura 5A). El indicador 
Bootstrap mean estimó 18 especies en CB y CM y 20 en CA (Figura 5B), con un esfuerzo de muestreo de 94, 91 y $86 \%$, respectivamente. La curva asintótica obtenida indica que la intensidad de muestreo fue adecuada (Álvarez et al. 2006).

En las tres condiciones de cobertura del dosel se encontró un total de 24 especies de herbáceas de 21 géneros y 12 familias, $50 \%$ nativas, $8 \%$ de ellas endémicas, $38 \%$ exóticas y $4 \%$ sin identificar. Las familias Asteraceae y Fabaceae presentaron la mayor riqueza, con seis y tres géneros, respectivamente. La abundancia de especies presentó una variación alta (1 a 1398 individuos; Cuadro 1). Diez de las 12 familias identificadas se encontraron en las tres condiciones de cobertura del dosel. En CB se registró el mayor número de especies, sin diferencias estadísticas significativas entre las condiciones de cobertura del dosel para forma de vida, riqueza total e índice de Margalef (Cuadro 2). Los valores para avena se presentan por separado para evitar una sobreestimación de la diversidad vegetal, especie utilizada como cultivo y de amplia distribución y cobertura en la Isla.

Los índices de Simpson, Fisher y Shannon-Wiener
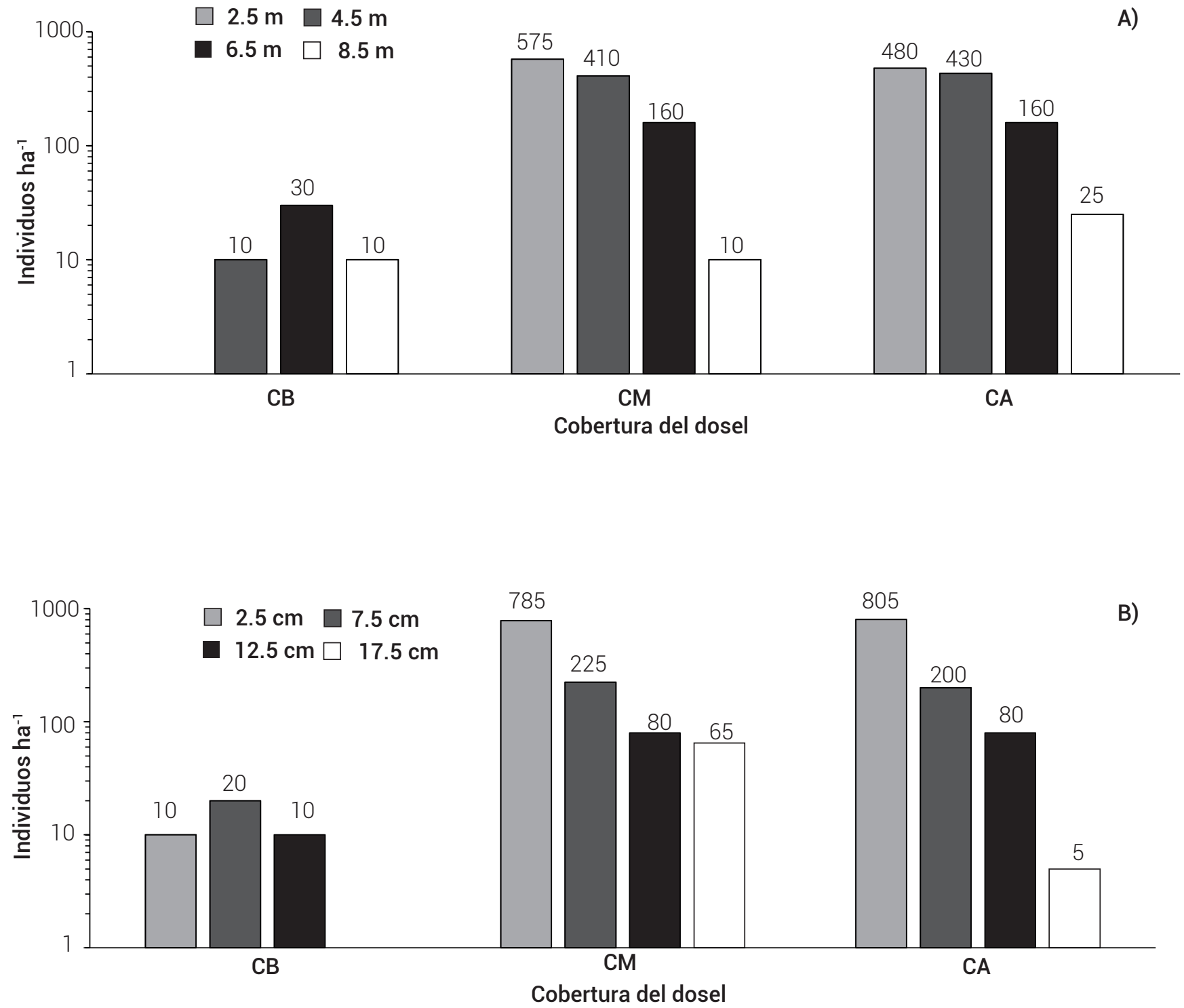

Figura 3. Número de juveniles (individuos $\mathrm{ha}^{-1}$ ) de $\mathrm{H}$. guadalupensis por $\mathrm{A}$ ) categoría de altura $(\mathrm{m})$ y $\mathrm{B}$ ) categoría diamétrica $(\mathrm{cm})$ en tres coberturas de dosel: baja (CB), media (CM) y alta (CA) en un rodal en recuperación en Isla Guadalupe, México. El número de individuos se presenta en escala logarítmica. 

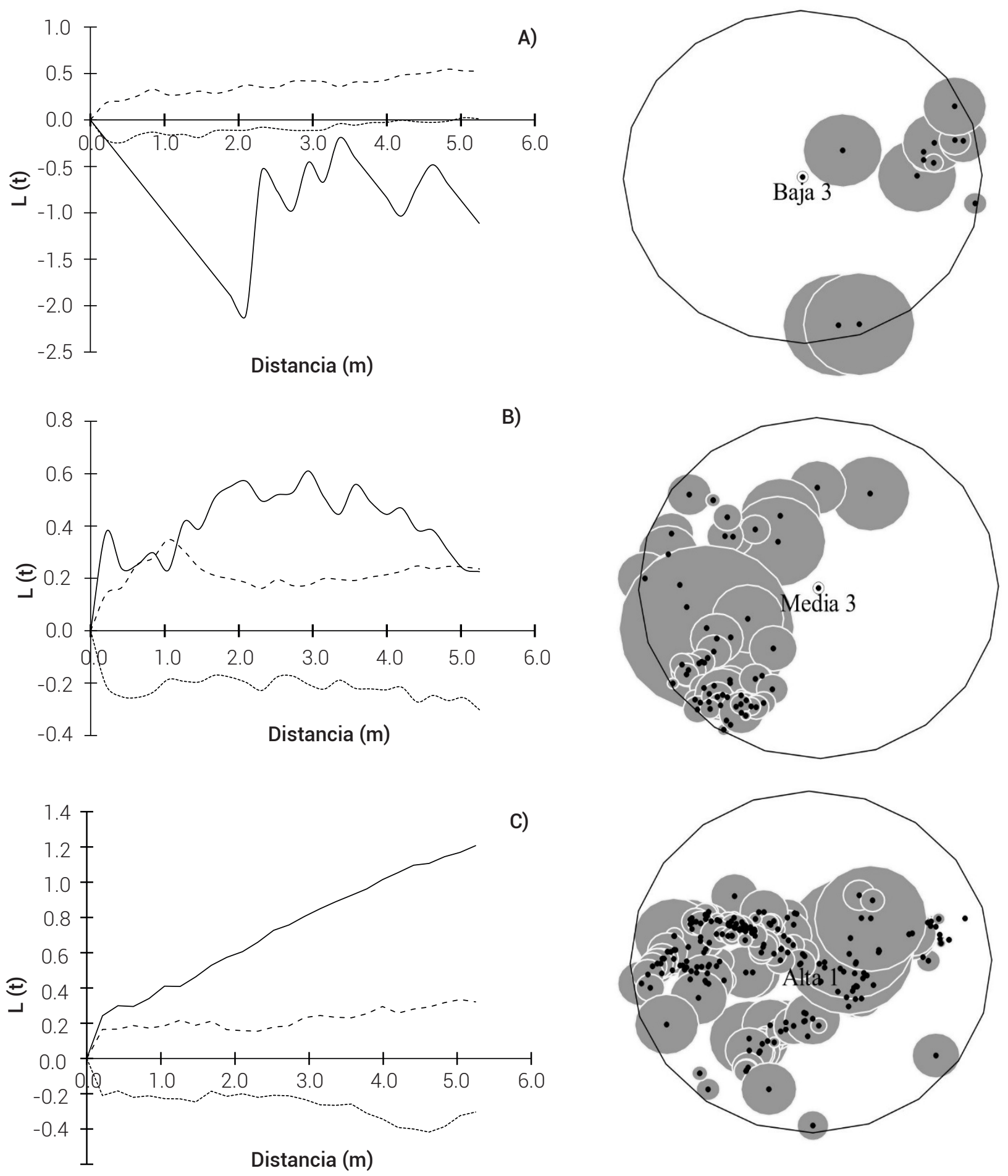

Figura 4. Índice de distribución espacial $\mathrm{L}_{(\mathrm{t})}$ de juveniles de $H$. guadalupensis en (A) sitio de $C B$, (B) CM, y (C) CA. Las líneas sólidas representan los valores de $\mathrm{L}(\mathrm{t})$, y las líneas discontinuas el intervalo de confianza con un $99 \%$ de confiabilidad. Del lado derecho se muestra la distribución espacial de juveniles (puntos) y la cobertura de copas de los adultos (círculos grises) en el sitio, en un rodal en recuperación en Isla Guadalupe, México 

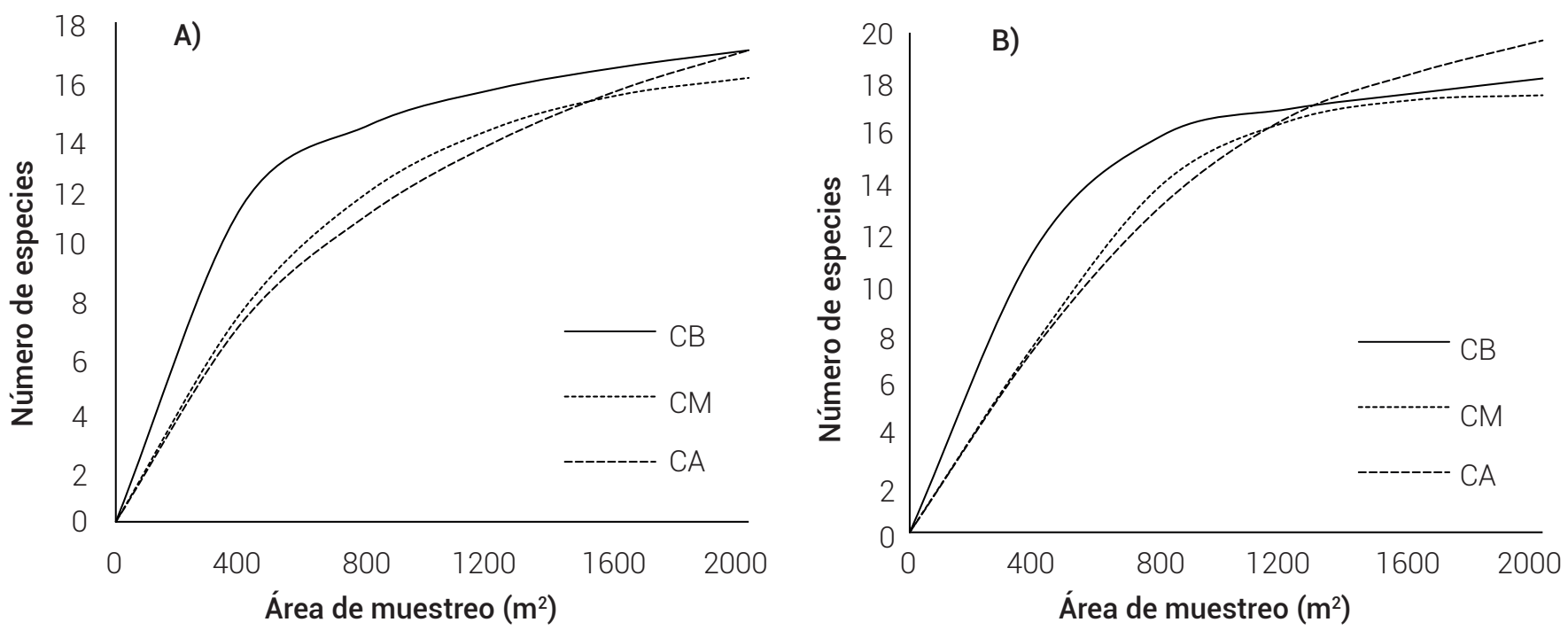

Figura 5. A) indicadores de S(est) y B) bootstrap mean en las curvas de acumulación de especies en tres condiciones de cobertura de dosel de $H$. guadalupensis: $\mathrm{CB}, \mathrm{CM}$ y CA. Las curvas de ACE mean y Chao son similares a las de $S$ (est).

fueron ligeramente mayores en la CB $(0.71,1.01$ y 1.55 , respectivamente). El índice de Simpson varió entre 0.61 y 0.71 en las tres condiciones de dosel, indicando una diversidad vegetal intermedia (valores cercanos a 1 indican mayor diversidad de la muestra) (Magurran, 2004).

El índice de Fisher indica la diversidad en función del número de individuos y del número de especies, y depende menos del tamaño del área de estudio (Condit et al., 1996). En el bosque de $H$. guadalupensis analizado, los valores encontrados de dicho índice variaron entre 0.76 y 1.01 , lo que representa una diversidad relativamente baja; por su parte, los valores del índice de Shannon-Wiener registrados en el presente estudio oscilaron entre 1.25 y 1.55. De acuerdo con Pla (2006), estos valores indican una diversidad baja. El 92\% del total de las especies encontradas en el sotobosque fueron herbáceas (Cuadro 1). Un mayor número y mayor abundancia de especies herbáceas que arbustivas podría indicar fases sucesionales tempranas; sin embargo, es difícil señalar con certeza el estado sucesional (Kimmins, 2004), ya que se carece de información de la estructura de las comunidades vegetales antes de la introducción de las cabras; lo que es evidente es la recuperación de áreas degradadas y un mayor número de especies herbáceas nativas. La mayor presencia de herbáceas puede ser explicada por el hecho de tratarse de una zona muy degradada, donde las primeras especies que se establecerán son las herbáceas, capaces de soportar condiciones climáticas adversas (Kimmins, 2004).
Alday et al. (2012) reportaron valores altos en el índice de diversidad de Shannon (> 4.2) en la regeneración natural de Pinus pinaster Ait, con una mayor riqueza en el estrato herbáceo, lo cual coincide con los resultados del presente estudio. La menor cobertura del dosel en CB origina áreas grandes con mayor exposición a la luz, lo cual fomenta el establecimiento y riqueza de especies, particularmente de herbáceas anuales.

Por su parte, el índice de Jaccard varió de 61 a 73 \% y el índice de Sørensen de 76 a 84 \% (Cuadro 3). La mayor similitud calculada, con ambos índices, fue entre CB y CA (72.7 y $84.2 \%$ ). Las tres condiciones de dosel comparten al menos $60 \%$ de las especies herbáceas registradas. El reducido número de especies y la superficie del área de estudio no permitieron detectar diferencias entre los niveles de cobertura; sin embargo, algunas especies fueron exclusivas de cada cobertura; por ejemplo, Centaurea melitensis y Lupinus niveus en CA, Senecio palmeri en CM y Calystegia macrostegia en CB (Cuadro 1).

Stoddard et al. (2011) encontraron que tratamientos de restauración basados en raleo del dosel y quema controlada favorecieron la aparición de especies nativas; sin embargo, después de seis años la composición de las comunidades evaluadas seguía cambiando, lo cual podría asemejarse a la condición actual del bosque de $\mathrm{H}$. guadalupensis. El área de estudio se eligió por no haber sido afectada por el incendio de 2008, condición que podría representar un cambio en la composición de las especies 
Cuadro 1. Riqueza y abundancia (número de individuos) de especies arbustivas y herbáceas en sitios de muestreo en tres condiciones de cobertura de dosel de $H$. guadalupensis.

\begin{tabular}{|c|c|c|c|}
\hline \multirow{2}{*}{ Familia } & \multicolumn{3}{|c|}{ Cobertura del dosel } \\
\hline & $\mathrm{CB}$ & $\mathrm{CM}$ & CA \\
\hline \multicolumn{4}{|l|}{ Asteraceae } \\
\hline Centaurea melitensis L. (herbácea) & - & - & 255 \\
\hline Lactuca serriola L. (herbácea) & 93 & 19 & 189 \\
\hline Logfia filaginoides (Hook. \& Arn.) Morefield (herbácea) & 292 & 389 & - \\
\hline Senecio palmeri (arbusto) & - & 6 & - \\
\hline Sonchus oleraceus L. (herbácea) & 1 & 10 & 69 \\
\hline Uropappus lindleyi (DC.) Nutt. (herbácea) & 114 & 54 & 143 \\
\hline \multicolumn{4}{|l|}{ Brassicaceae } \\
\hline Sisymbrium sp. L. (herbácea) & 16 & 3 & 9 \\
\hline \multicolumn{4}{|l|}{ Caryophyllaceae } \\
\hline Herniaria hirsuta L. var. cinerea (DC.) Loret \& Barrandon (herbácea) & 1398 & 43 & - \\
\hline Silene gallica L. (herbácea) & 536 & 375 & 136 \\
\hline \multicolumn{4}{|l|}{ Convolvulaceae } \\
\hline $\begin{array}{l}\text { Calystegia macrostegia (Greene) Brummitt ssp. macrostegia (herbácea perenne, } \\
\text { enredadera) }\end{array}$ & 4 & - & - \\
\hline \multicolumn{4}{|l|}{ Fabaceae } \\
\hline Acmispon grandiflorus (Benth.) Brouillet var. grandiflorus (herbácea perenne) & 1 & - & 3 \\
\hline Lupinus niveus S.Watson (arbusto) & - & - & 6 \\
\hline Trifolium microcephalum Pursh (herbácea) & 107 & 4 & 5 \\
\hline Trifolium palmeri S. Watson (herbácea) & 16 & - & 5 \\
\hline \multicolumn{4}{|l|}{ Geraniaceae } \\
\hline Erodium cicutarium (L.) Aiton (herbácea) & 1044 & 107 & 2 \\
\hline \multicolumn{4}{|l|}{ Malvaceae } \\
\hline Sphaeralcea sulphurea S. Watson (arbusto) & 478 & 37 & 9 \\
\hline \multicolumn{4}{|l|}{ Montiaceae } \\
\hline Calandrinia menziesii (Hook.) Torr. \& A. Gray (herbácea) & 85 & 31 & 5 \\
\hline Claytonia sp. (herbácea) & - & 24 & 87 \\
\hline \multicolumn{4}{|l|}{ Poaceae } \\
\hline Avena sp. (herbácea) (valores en cobertura, \%) & 21.3 & 17.4 & 20.7 \\
\hline \multicolumn{4}{|l|}{ Polemoniaceae } \\
\hline Gilia nevinii A. Gray (herbácea) & 821 & 233 & 52 \\
\hline \multicolumn{4}{|l|}{ Polygonaceae } \\
\hline Pterostegia drymarioides Fischer. \& C.A. Mey. (herbácea) & 150 & 60 & 303 \\
\hline \multicolumn{4}{|l|}{ Themidaceae } \\
\hline Dichelostemma capitatum (Benth.) Alph. Wood ssp. capitatum (herbácea) & 66 & - & 226 \\
\hline No identificado (herbácea) (valores en cobertura, \%) & - & - & 0.25 \\
\hline
\end{tabular}


Cuadro 2. Valores promedio de riqueza por forma de vida y total e índice de riqueza de Margalef en tres niveles de cobertura de dosel de $H$. guadalupensis: $\mathrm{CB}, \mathrm{CM}$ y $\mathrm{CA}$.

\begin{tabular}{|c|c|c|c|c|c|}
\hline $\begin{array}{l}\text { Cobertura del } \\
\text { dosel }\end{array}$ & Arbustos & Herbáceas & Avena & Riqueza total & Índice de Margalef \\
\hline $\mathrm{CB}$ & $0.80 \pm 0.20 a$ & $10.60 \pm 0.51 \mathrm{a}$ & $2.00 \pm 0.00 \mathrm{a}$ & $13.40 \pm 0.50 \mathrm{a}$ & $0.92 \pm 0.04 a$ \\
\hline $\mathrm{CM}$ & $0.60 \pm 0.40 a$ & $7.00 \pm 1.34 \mathrm{a}$ & $2.00 \pm 0.00 \mathrm{a}$ & $9.60 \pm 1.69 \mathrm{a}$ & $0.67 \pm 0.13 \mathrm{a}$ \\
\hline CA & $0.60 \pm 0.25 a$ & $6.60 \pm 1.63 \mathrm{a}$ & $2.00 \pm 0.00 \mathrm{a}$ & $9.20 \pm 1.62 \mathrm{a}$ & $0.68 \pm 0.17 a$ \\
\hline
\end{tabular}

Valores promedio en las columnas seguidos de la misma letra no son estadísticamente diferentes ( $\leq \leq 0.05)$. CB: cobertura baja, CM: cobertura media, CA: cobertura alta.

Cuadro 3. Índices de Jaccard y Sørensen (diversidad beta) del sotobosque de $H$. guadalupensis en tres categorías de cobertura de dosel.

\begin{tabular}{|c|c|c|c|c|c|}
\hline h & Cober & $\begin{array}{l}\text { Especies en } \\
\text { Cobertura } 1\end{array}$ & $\begin{array}{l}\text { Especies en } \\
\text { Cobertura } 2\end{array}$ & $\begin{array}{c}\text { Especies } \\
\text { compartidas }\end{array}$ & $\begin{array}{l}\text { Índice de } \\
\text { Sørensen (\%) }\end{array}$ \\
\hline
\end{tabular}

\begin{tabular}{lllllll}
\hline $\mathrm{CB}$ & $\mathrm{CM}$ & 19 & 18 & 15 & 68.18 & 81.08 \\
$\mathrm{CB}$ & $\mathrm{CA}$ & 19 & 19 & 16 & 72.73 & 84.21 \\
$\mathrm{CM}$ & $\mathrm{CA}$ & 18 & 19 & 14 & 60.87 & 75.68 \\
\hline
\end{tabular}

CB: cobertura baja, CM: cobertura media, CA: cobertura alta.

que se desarrollan en el sotobosque de las diferentes coberturas. A partir de la erradicación de las cabras en toda la isla se ha reportado la aparición de especies que se creían extintas o extirpadas y que han recolonizado espacios anteriormente ocupados por especies exóticas (Ceceña-Sánchez et al., 2018).

\section{CONCLUSIONES}

Los resultados muestran un aumento evidente en el número de individuos juveniles y de plántulas en el bosque de $H$. guadalupensis después de la erradicación de las cabras. La cobertura de dosel no ha tenido un efecto significativo en la cantidad de los primeros, pero sí en los segundos, asociada con la disponibilidad de semilla y la protección del dosel contra factores ambientales adversos. La estructura vertical y horizontal de los individuos juveniles se asocia principalmente con una estructura típica de reclutamiento gradual y continuo en la recuperación de superficie y rejuvenecimiento del bosque. La distribución espacial sigue un patrón común relacionado con la apertura de claros en el dosel que fomentan el establecimiento de nuevos individuos en forma agregada. La diversidad del sotobosque es similar en los tres niveles de cobertura del dosel y hay un porcentaje elevado (65-70 \%) de especies comunes entre ellas. La vegetación herbácea presenta la mayor riqueza de especies y abundancia de individuos que la vegetación arbustiva. Se muestra una notable recuperación de la diversidad vegetal en el área de estudio, con la presencia de al menos $58 \%$ de especies endémicas o nativas de la región, sin que hasta el momento se haya detectado un efecto de la cobertura del dosel o interferencia de los individuos juveniles de $H$. guadalupensis.

\section{AGRADECIMIENTOS}

Al Grupo de Ecología y Conservación de Islas por el apoyo logístico en la fase de campo. A la CONANP-Reserva de la Biosfera Isla Guadalupe por permitir el acceso a la zona. A la Secretaría de Marina (SEMAR) por el apoyo en el uso de sus embarcaciones.

\section{BIBLIOGRAFÍA}

Aguirre-Muñoz A., A. Samaniego-Herrera, L. Luna-Mendoza, A. Ortiz-Alcaraz, M. Rodríguez-Malagón, M. Félix-Lizárraga, ... and M. Latofski-Robles (2011) Island restoration in Mexico: ecological outcomes after a decade of eradications of invasive mammals. In: Island Invasives: Eradication and Management. C. R. Veitch, M. N. Clout and D. R. Towns (eds.). International Union for Conservation of Nature. Gland, Switzerland. pp:250-258.

Alday J. G., C. Martínez y F. Bravo (2012) Regeneración natural y conservación de la diversidad vegetal en masas de Pinus pinaster Ait. In: La Regeneración Natural de los Pinares en los Arenales de la Meseta Castellana. J. Gordon, M. Calama, M. Pardos, F. Bravo y G. Montero (eds.). Instituto Universitario de Investigación en Gestión Forestal Sostenible, Universidad de 
Valladolid-INIA. Valladolid, España. pp:219-235

Álvarez M., S. Córdoba, F. Escobar, G. Fagua, F. Gast, H. Mendoza, ... and H. Villarreal (2006) Manual de Métodos para el Desarrollo de Inventarios de Biodiversidad. Programa de Inventarios de Biodiversidad. Instituto de investigación de Recursos Biológicos Alexander von Humboldt. Bogotá, Colombia. 235 p.

Besag J. (1977) Contribution to the discussion on Dr Ripley's paper Journal of the Royal Society B (Methodological) 39:193-195, https://doi.org/10.1111/j.2517-6161.1977.tb01616.x

Ceceña-Sánchez M. L., A. Aguirre-Muñoz y J. Delgadillo-Rodríguez (2018) Plant scrubs associations of Guadalupe Island Mexico. Polibotánica 46:49-70, https://doi.org/10.18387/ polibotanica.46.3

Chao A. (1984) Nonparametric estimation of the number of classes in a population. Scandinavian Journal of Statistics 11:265-270.

Chao A. and S. M. Lee (1992) Estimating the number of classes via sample coverage. Journal of the American Statistical Association 87:210-217, https://doi.org/10.2307/2290471

CONANP, Comisión Nacional de Áreas Naturales Protegidas (2013) Programa de Manejo Reserva de la Biosfera Isla Guadalupe. Secretaría de Medio Ambiente y Recursos Naturales. Mexico, D.F. 232 p.

Condit R., S. P. Hubble, J. V. Lafrankie, R. Sukumar, N. Manokaran, R. B. Foster and M. S. Ashton (1996) Species-area and species-individual relationships for tropical trees: a comparison of three 50-ha plots. Journal of Ecology 84:549-562.

Corral-Rivas J. J., B. Vargas-Larreta, C. Wehenkel, 0. A. Aguirrre-Calderón y F. Crecente-Campo (2013) Guía para el Establecimiento, Seguimiento y Evaluación de Sitios Permanentes de Monitoreo en Paisajes Productivos Forestales. Fondo Sectorial para la Investigación, el Desarrollo y la Innovación Tecnológica Forestal. CONACYT-CONAFOR. México, D. F. 93 p

Fisher M. (2000) Toolbos: Software Available for Sophisticated Spatial Statistics. University of South Pacifc. Suva, Fiji. 28 p.

García E. (2004) Modificaciones al Sistema de Clasificación Climática de Köppen. 5a edición. Instituto de Geografía, Universidad Nacional Autónoma de México. México, D. F. 90 p.

Garcillán P. P. (2010) Seed release without fire in Callitropsis guadalupensis, a serotinous cypress of a Mediterraneanclimate oceanic island. Journal of Arid Environments 74:512 515, https://doi.org/10.1016/j.jaridenv.2009.09.017

Garcillán P. P., E. Vega and E. López-Reyes (2009) Recruitment response of Guadalupe cypress (Callitropsis guadalupensis) three years after goat eradication on Guadalupe island. In: Proceedings of the 7th California Islands Symposium. C. C. Damiani and D. K. Garcelon (eds.). Institute for Wildlife Studies. Arcanta, California, USA. pp:177-183.

Greig-Smith P. (1983) Quantitative Plant Ecology. University of California Press. Berkeley, California, USA. 359 p.

Hammer $\emptyset$., D. A. T. Harper and P. D. Ryan. (2020) PAST: Paleontological statistics software package for education and data analysis. Paleontología Electrónica 4:1-9.

Junak S., K. Bradford, B. Tershy, D. Croll, L. M. Luna-Mendoz y A. AguirreMuñoz (2005) Esfuerzos recientes de conservación y apuntes sobre el estado actual de la flora de Isla Guadalupe In: Isla Guadalupe, Restauración y Conservación. K. Santos y E. Peters (comps.). Instituto Nacional de Ecología. México, D. F. pp:83-93

Kimmins J. P. (2004) Forest Ecology: A Foundation for Sustainable Management. Prentice Hall. Hoboken, New Jersey, USA. 596 p.

León L. J. L., J. P. Rebman and T. Oberbauer (2003) On the urgency of conservation on Guadalupe Island, Mexico: is it a lost paradise? Biodiversity and Conservation 12:1073-1082, https://doi org/10.1023/A:1022854211166

Luna-Mendoza L., A. Aguirre-Muñoz, J. C. Hernández-Montoya, M. TorresAguilar, J. S. García-Carreón, O. Puebla-Hernández, ... and F. MéndezSánchez (2019) Ten years after feral goat eradication: the active restoration of plant communities on Guadalupe Island, Mexico. In: Island Invasives: Scaling up to Meet the Challenge. C. R. Veitch, M. N. Clout, A. R. Martin, J. C. Russell and C. J. West (eds.). IUCN Occasional Paper SSC No. 62. International Union for Conservation of Nature and Natural Resources. Gland Switzerland. pp:571-575.

Maciel-Nájera J. F., J. Hernández-Velasco, M. S. González-Elizondo, J. C. Hernández-Díaz, C. A. López-Sánchez, P. Antúnez, ... and C. Wehenkel (2020) Unexpected spatial patterns of natural regeneration in typical uneven-aged mixed pine-oak forests in the Sierra Madre Occidental, Mexico. Global Ecology and Conservation 23:e01074, https://doi.org/10.1016/j.gecco.2020.e01074

Magurran A. E. (2004) Measuring Biological Diversity. Blackwell Publishing. Oxford, UK. 256 p.

Moran R. (1996) The Flora of Guadalupe Island, Mexico. Issue 19 Memoirs of the California Academy of Sciences. San Francisco, California, USA. $190 \mathrm{p}$

Moreno C. E. (2001) Métodos para Medir la Biodiversidad. M\&T Manuales y Tesis SEA. Vol. 1. Zaragoza, España. 84 p.

Oberbauer T. A. (2006) La vegetación de Isla Guadalupe. Entonces y ahora. Gaceta Ecológica 81:47-58.

Pla L. (2006) Biodiversidad: inferencia basada en el índice de Shannon y la riqueza. Interciencia 31:583-590.

Plateros-Gastélum P. A., V. J. Reyes-Hernández, A. Velázquez-Martínez, P. Hernández-de la Rosa y G. V. Campos-Ángeles (2018) Disponibilidad de luz bajo dosel en rodales de Abies religiosa. Madera y Bosques 24:e2431711, https://doi.org/10.21829/myb.2018.2431711 https://doi.org/10.21829/myb.2018.2431711

Pommerening A. and P. Grabarnik (2019) Individual-based Methods in Forest Ecology and Management. Springer. Cham, Switzerland. $411 p$

R Core Team (2019) R: A Language and Environment for Statistical Computing. R Foundation for Statistical Computing, Vienna, Austria. URL https://www.R-project.org/ (November 2020).

Ripley B. D. (1977) Modelling spatial patterns. Journal of the Royal Statistical Society, Series B (Methodological) 39:172-192, https://doi.org/10.1111/j.2517-6161.1977.tb01615.x

Rodríguez-Laguna R., R. Razo-Zárate, J. Fonseca-González, J. CapulínGrande y R. Goche-Telles (2015) Regeneración natural postincendio de Abies religiosa (H.B.K.) Schl. et Cham, en el Parque Nacional "El Chico" Hidalgo. Revista Iberoamericana 2:11-22

Ruiz-Jaén M. C. and T. M. Aide (2005) Vegetation structure, species diversity, and ecosystem processes as measures of restoration success. Forest Ecology and Management 218:159-173, https://doi.org/10.1016/j.foreco.2005.07.008

SEMARNAT, Secretaría del Medio Ambiente y Recursos Naturales (2010) Norma Oficial Mexicana NOM-059-SEMARNAT-2010, Protección ambiental-Especies nativas de México de flora y fauna silvestres-Categorías de riesgo y especificaciones para su inclusión, exclusión o cambio-Lista de especies en riesgo. Diario Oficial de la Federación. Ciudad de México. 10 de diciembre de 2010

Smith E. P. and G. Van Belle (1984) Nonparametric estimation of species richness. Biometrics 40:119-129, https://doi org $/ 10.2307 / 2530750$

Spies T. A. (1998) A key to the ecosystem. In: Proceedings of a Workshop on Structure, Process, and Diversity in Successional Forests of Coastal British Columbia. J. A. Trofymow and A. MacKinnon (eds.). Northwest Science. Victoria, British Columbia, Canada pp:34-39.

Stoddard M. T., C. M. McGlone, P. Z. Fulé, D. C. Laughkin and M. L. Daniels (2011) Native plants dominate understory vegetation following ponderosa pine forest restoration treatments. Western North American Naturalist 71:206-214, https://doi org/10.3398/064.071.0207

Toledo-López J. F., J. I. Valdez-Hernández, M. A. Pérez-Farrera y V. M. CetinaAlcalá (2012) Composición y estructura arbórea de un bosque tropical estacionalmente seco en la Reserva de la Biósfera La Sepultura, Chiapas. Revista Mexicana de Ciencias Forestales 3:43-56.

Ustin S. L., R. A. Woodward, M. G. Barbour and J. L. Hatfield (1984) Relationships between sunfleck dynamics and red fir seedling distribution. Ecology 65:1420-1428, https://doi. org/10.2307/1939122

Ventura-Ríos A., F. O. Plascencia-Escalante, P. Hernández R., G. ÁngelesPérez y A. Aldrete (2017) ¿Es la reforestación una estrategia para la rehabilitación de bosques de pino? Una experiencia en el centro de México. Bosque 38:55-66, https://doi.org/10.4067/ S0717-92002017000100007

Way D. A. and R. W. Pearcy (2012) Sunflecks in trees and forests: from photosynthetic physiology to global change biology. Tree Physiology 32:1066-1081, https://doi.org/10.1093/treephys/ tps064 
Zarco-Espinoza V. M., J. I. Valdez-Hernández, G. Ángeles-Pérez y 0. CastilloAcosta (2010) Estructura y diversidad de la vegetación arbórea del Parque Estatal Agua Blanca, Macuspana, Tabasco. Universidad y Ciencia Trópico Húmedo 26:1-17. 